\title{
A Large Scale System Model-Order Reduction Method Based on SVD-Krylov
}

\author{
Zhe Yan, Fangming Lu and lin Zhou \\ Zhe Yan is a professor at the department of automation from Harbin University of \\ Science and Technology. His research area mainly includes control theory and \\ applications. \\ yanzhehrb@163.com
}

\begin{abstract}
A SVD-Krylov method for large scale MIMO(multi-input multi-output) system model-order reduction is proposed in this paper. Its aim is to combined the singular value decomposition(SVD)and Krylov methods by retaining the best feature that can be applied for solving some problems for the large scale system model-order reduction. The method matches the first $r$ Markov parameters and minimizes the error in the remaining ones in the least-squares sense. The reduced model is asymptotically stable, matches a certain number of moments, and minimizes a weighted error in the discrete time case. The effectiveness of the proposed approaches is tested by the Iss (international space station)model that in the SLICOT library, getting the frequency-response, the error and the error bounds of different order reduced model. The result shows that the proposed method is efficiently.
\end{abstract}

Keywords: model order reduction; SVD-Krylov method; moment matching

\section{Introduction}

With the development of science and technology, in fluid dynamics, the controller design, mechanical engineering, aerospace engineering, integrated circuits and other fields need to build the higher order model . Calculation of high order model equations of the particularly difficult and complicated, it is difficult to use traditional analysis methods to ensure that the analysis of the model is accurate and efficient [1]. Therefore, in order to solve this problem, the high order model order reduction analysis has become the inevitable trend. Model order reduction is a high order model of the original large system using low order model to approximate, and keep the original system's main features, such as the stability of the system and dynamic process [2].

There are many different model order reduction methods. The first one is Multi pointfit model reduction [3]. This method for nonlinear system model-order reduction is efficiently and mainly applied to the block Arnoldi algorithm and Frobeniu norm. The second is Krylov subspace method [4]. This method mainly includes classic Arnoldi and Lanczos order reduction. Krylov based model reduction methods are based on matching the so called moments of the original transfer function around some selected frequencies [5-6]. The last is SVD model order reduction method [7-8]. In this SVD based model reduction [9], Hankel singular values play the role of the singular values in approximation of constant matrices [10]. One of the most common approaches in this category is Balanced Model Reduction. The methods belong to a kind of single order reduction method, which already could not meet the requirements of order reduction. This paper combines the traditional Krylov method with SVD method, This method not only can keep Krylov subspace model order reduction method of numerical analysis advantages, but with the theoretical characteristics of the SVD method, like stability and the global 
error bounds. An illustrating example of international space station for the large scale system model-order reduction is given to verify the SVD-Krylov method order reduction effect.

\section{The Basic Theory of Krylov Model Order Reduction Method}

Many phenomena are modeled with linear time-invariant systems, given a state-space of large-scale MIMO system.

$$
\left\{\begin{array}{l}
\mathbf{E} \dot{\mathbf{x}}(t)=\mathbf{A x}(t)+\mathbf{B u}(t) \\
\dot{\mathbf{y}}(t)=\mathbf{C x}(t)+\mathbf{D u}(t)
\end{array}\right.
$$

With $\mathbf{E}, \mathbf{A} \in R^{n \times n}, \mathbf{B} \in R^{n \times p}, \mathbf{C} \in R^{m \times n}$. The state vector $\mathbf{x}(t) \in R^{n}$, the scalar input $\mathbf{u}(t) \in R^{p}$ and the scalar output $\mathbf{y}(t) \in R^{m}$.be expressed as:

$$
\Sigma:=\left(\begin{array}{c:c}
\mathbf{A} & \mathbf{B} \\
\hdashline \mathbf{C} & \mathbf{D}
\end{array}\right)
$$

To simplify the calculation, assumed that the system initial state is 0 , given the transfer function of the original system.

$$
\mathbf{H}(\mathrm{s})=\mathbf{C}(\mathbf{A}-s \mathbf{E})^{-1} \mathbf{B}
$$

The transfer function of Taylor series expansion in the $s_{0}[11]$.

$$
\mathbf{H}(\mathrm{s})=-\mathbf{C}\left(\mathbf{A}-s_{0} \mathbf{E}\right)^{-1} \mathbf{B}-\cdots-\mathbf{C}\left(\left(\mathbf{A}-s_{0} \mathbf{E}\right)^{-1} \mathbf{E}\right)^{i}\left(\mathbf{A}-s_{0} \mathbf{E}\right)^{-1} \mathbf{B}\left(s-s_{0}\right)^{i}-\cdots
$$

Thus can be obtained the $i$ th order moments of the transfer function in the $s_{0}$.

$$
\begin{aligned}
& \mathbf{M}_{i}=\mathbf{C}\left(\left(\mathbf{A}-s_{0} \mathbf{E}\right)^{-1} \mathbf{E}\right)^{i}\left(\mathbf{A}-s_{0} \mathbf{E}\right)^{-1} \mathbf{B}\left(s-s_{0}\right)^{i} \\
& \mathbf{H}(s)=\sum_{j=0}^{\infty} \eta_{s_{0}}^{(j)}\left(s_{0}-s\right)^{j}
\end{aligned}
$$

In the eig.6, the $\eta_{s_{0}}^{(j)}$ is the $j$ th pattern in the $s_{0}(j \geq 0)$. In the case of $s_{0} \neq \infty$.

$\eta_{s_{0}}^{(j)}=\mathbf{C}\left(s_{0} \mathbf{E}-\mathbf{A}\right)^{-(j+1)} \mathbf{B}$

In the case of $s_{0}=\infty$.

$\eta_{s_{0}}^{(0)}=0, \quad \eta_{s_{0}}^{(j)}=C A^{j-1} B(1-)$

The key of Krylov order reduction method is model matching approach. First of all is to find a reduced order model $\Sigma_{r}$. The transfer function is [12].

$$
\mathbf{H}_{r}(s)=\sum_{j=0}^{\infty} \hat{\eta}_{s_{0}}^{(j)}\left(s_{0}-s\right)^{j}
$$

The transfer function must ensure that the parts of the model can be exactly equal with the parts of the original transfer function. In other words.

$$
\eta_{s_{0}}^{(j)}=\hat{\eta}_{s_{0}}^{(j)}, j=0,1,2, \cdots, k, \cdots
$$




\section{The SVD Model Order Reduction Method}

In this thesis, we will interested in the linear time invariant systems described in state space form.

$$
\left\{\begin{array}{l}
\dot{\mathbf{x}}(t)=\mathbf{A x}(t)+\mathbf{B u}(t) \\
\dot{\mathbf{y}}(t)=\mathbf{C x}(t)+\mathbf{D u}(t)
\end{array} \quad \boldsymbol{\Sigma}:=\left(\begin{array}{c:c}
\mathbf{A} & \mathbf{B} \\
\hdashline \mathbf{C} & \mathbf{D}
\end{array}\right)\right.
$$

The system is observably and controllably, if this system is met [13].

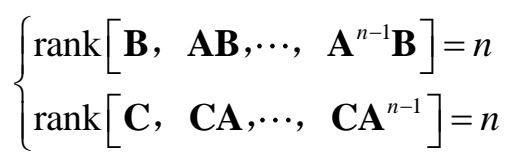

There are two important Lyapunov equations in this system.

$$
\left\{\begin{array}{l}
\mathbf{A P}+\mathbf{P} \mathbf{A}^{\mathrm{T}}+\mathbf{B} \mathbf{B}^{\mathrm{T}}=0 \\
\mathbf{A}^{\mathrm{T}} \mathbf{Q}+\mathbf{Q A}+\mathbf{C}^{\mathrm{T}} \mathbf{C}=0
\end{array}\right.
$$

Assumed that the system is stable, the observable and controllable Gramians matrixes $\mathbf{P}$ and $\mathbf{Q}$ can be calculated by use the Lyapunov matrix equation. $\mathbf{P}$ and $\mathbf{Q}$ are positive definite matrixes.

$$
\begin{aligned}
\mathbf{P} & :=\int_{0}^{\infty} e^{\mathbf{A} \tau} \mathbf{B} \mathbf{B}^{\mathrm{T}} e^{\mathbf{A}^{\mathrm{T}} \tau} d \tau \\
\mathbf{Q} & :=\int_{0}^{\infty} e^{\mathbf{A}^{\mathrm{T}} \tau} \mathbf{C}^{\mathrm{T}} \mathbf{C} e^{\mathbf{A} \tau} d \tau
\end{aligned}
$$

Cholesky decomposition performed on the observable and controllable $\mathbf{P}$ and $\mathbf{Q}$, $\mathbf{P}=\mathbf{C}_{0} \mathbf{C}_{0}^{\mathrm{T}}, \quad \mathbf{Q}=\mathbf{O}_{b} \mathbf{O}_{b}^{\mathrm{T}}$. Finding a transformation matrix $\mathbf{T}$ and satisfy the following conditions.

$$
\left\{\begin{array}{l}
\overline{\mathbf{P}}=\mathbf{T P T}^{\mathrm{T}} \\
\overline{\mathbf{Q}}=\left(\mathbf{T}^{-1}\right)^{\mathrm{T}} \mathbf{Q} \mathbf{T}^{-1}
\end{array}\right.
$$

For the linear system, if the observable and controllable Gramians are equality. Obtain-ed the $\mathbf{C}_{0}^{\mathrm{T}} \mathbf{O}_{b}=\mathbf{U} \boldsymbol{\Sigma} \mathbf{V}^{\mathrm{T}}$ by decomposed the $\mathbf{C}_{0}^{\mathrm{T}} \mathbf{O}_{b}$ using the SVD method. Where $\mathbf{U}$ and $\mathbf{V}$ are orthogonal matrix, $\Sigma$ is a diagonal matrix [14].

$$
\boldsymbol{\Sigma}=\tilde{\mathbf{P}}=\tilde{\mathbf{Q}}=\left[\begin{array}{cccc}
\sigma_{1} & 0 & \cdots & 0 \\
0 & \sigma_{2} & \cdots & 0 \\
\vdots & \vdots & \cdots & 0 \\
0 & 0 & \cdots & \sigma_{n}
\end{array}\right]
$$

$\sigma_{1} \geq \sigma_{2} \geq \sigma_{3} \cdots \geq \sigma_{n} \geq 0$ in the eig. (17), $\sigma_{i}$ is the Hankel singular values of the system ,the balanced truncation method can choose different length [14-15] of singular value of system. $\sigma_{1}$ is the maximum singular value.

$$
\|\Sigma\|_{\mathrm{H}}=\sqrt{\lambda_{\max }(\mathbf{P Q})}=\sigma_{1}
$$

The left and right eigenvector of the eigenvalues are $\mathbf{V}_{L}$ and $\mathbf{V}_{R}$. 


$$
\left\{\begin{array}{l}
\mathbf{V}_{\mathrm{L}}^{\mathrm{T}} \mathbf{P} \mathbf{Q}=\operatorname{diag}\left\{\sigma_{1}^{2}, \cdots, \sigma_{n}^{2}\right\} \mathbf{V}_{\mathrm{L}}^{\mathrm{T}} \\
\mathbf{P} \mathbf{Q} \mathbf{V}_{\mathrm{R}}=\mathbf{V}_{\mathrm{R}} \operatorname{diag}\left\{\sigma_{1}^{2}, \cdots, \sigma_{n}^{2}\right\}
\end{array}\right.
$$

$\mathbf{V}_{\mathrm{L}}=\left[\mathbf{V}_{\mathrm{Lmax}}, \mathbf{V}_{\mathrm{Lmin}}\right], \mathbf{V}_{\mathrm{R}}=\left[\mathbf{V}_{\mathrm{Rmax}}, \mathbf{V}_{\mathrm{R} \min }\right]$, The first $q$ large eigenvalues of left (right) of the corresponding eigenvectors constituted $\mathbf{V}_{\mathrm{L} \max }\left(\mathbf{V}_{\mathrm{R} \max }\right)$, the others eigenvalues of the residual form the left (right) eigenvector matrix $\mathbf{V}_{\mathrm{Lmin}}\left(\mathbf{V}_{\text {Rmin }}\right)$.

$$
\mathbf{E}_{\max }=\mathbf{U}_{\text {Emax }} \boldsymbol{\Sigma}_{\text {Emax }} \mathbf{V}_{\text {Emax }}^{\mathrm{T}}
$$

Where $\mathbf{E}_{\max }=\mathbf{V}_{\text {Lmax }}^{\mathrm{T}} \mathbf{V}_{\mathrm{Rmax}}^{\mathrm{T}}$

$$
\begin{aligned}
& \mathbf{S}_{\mathrm{Lmax}}=\mathbf{V}_{\mathrm{Lmax}} \mathbf{U}_{\mathrm{Emax}} \boldsymbol{\Sigma}_{\text {Emax }}^{-\frac{1}{2}} \mathbf{P}_{\mathrm{Lmax}}^{-\frac{1}{2}} \in R^{n \times q} \\
& \mathbf{S}_{\mathrm{R} \max }=\mathbf{V}_{\mathrm{R} \max } \mathbf{U}_{\text {Emax }} \boldsymbol{\Sigma}_{\text {Emax }}^{-\frac{1}{2}} \in R^{n \times q}
\end{aligned}
$$

The original system reduced to order $q$

$$
\left\{\begin{array}{l}
\tilde{\mathbf{A}}=\mathbf{S}_{\mathrm{Lmax}}^{\mathrm{T}} \mathbf{A} \mathbf{S}_{\mathrm{R} \max } \\
\tilde{\mathbf{B}}=\mathbf{S}_{\mathrm{Lmax}}^{\mathrm{T}} \mathbf{B} \\
\tilde{\mathbf{C}}=\mathbf{C S}_{\mathrm{R} \max }
\end{array}\right.
$$

The error bound of reduction-order is given by the Hankel singular value

$$
\left\|\boldsymbol{\Sigma}-\boldsymbol{\Sigma}_{\text {red }}\right\| \mathbf{H}_{\infty} \leq 2\left(\sigma_{k+1}+\cdots+\sigma_{n}\right)
$$

\section{The SVD-Krylov Model Order Reduction Method}

The SVD-Krylov method can combine the stability and error bound character of the SVD with the numerical calculations of the effective combination of Krylov, from that can improve the reduction efficient. Calculating the Hankel singular values of the system $\Sigma$, obtained.

$$
\mathbf{H}:=\left[\begin{array}{cccc}
\eta_{0} & \eta_{1} & \eta_{2} & \cdots \\
\eta_{1} & \eta_{2} & \eta_{3} & \cdots \\
\eta_{2} & \eta_{3} & \eta_{4} & \cdots \\
\vdots & \vdots & \vdots & \ddots
\end{array}\right]
$$

Assumed that the system is stably, The observable and controllable Gramians $\mathbf{P}$ and $\mathbf{Q}$ are

$$
\begin{aligned}
\mathbf{P} & :=\left[\begin{array}{lllll}
\mathbf{B} & \mathbf{A B} & \cdots & \mathbf{A}^{r-1} \mathbf{B} & \cdots
\end{array}\right] \\
\mathbf{Q} & :=\left[\begin{array}{lllll}
\mathbf{C}^{T} & \mathbf{A}^{T} \mathbf{C}^{T} & \cdots & \left(\mathbf{A}^{T}\right)^{r-1} \mathbf{C}^{T} & \cdots
\end{array}\right]^{T}
\end{aligned}
$$

In the eig. (25), the $\eta_{i}:=\mathbf{C A}^{i} \mathbf{B}$ is the $\mathrm{i}+1$ th parameter.

$$
\mathbf{H}=\mathbf{Q P}
$$


The first $r$ columns and the $r+1$ column of the Hankel singular values matrix are respectively denoted by $\mathbf{H}_{r}$ and $\mathbf{h}_{r}$ [16].

$$
\begin{gathered}
\mathbf{H}_{r}:=\left[\begin{array}{cccc}
\eta_{0} & \eta_{1} & \cdots & \eta_{r-1} \\
\eta_{1} & \eta_{2} & \cdots & \eta_{r} \\
\vdots & \vdots & \vdots & \vdots \\
\eta_{r} & \eta_{r+1} & \cdots & \eta_{2 r-1} \\
\vdots & \vdots & \vdots & \vdots
\end{array}\right] \\
\mathbf{h}_{r}=\left[\begin{array}{c}
\eta_{r} \\
\eta_{r+1} \\
\vdots \\
\eta_{2 r-1} \\
\vdots
\end{array}\right]
\end{gathered}
$$

The first $r$ columns of the observable and controllable Gramians $\mathbf{P}$ and $\mathbf{Q}$ are selected to constitute the new observable and controllable Gramians $\mathbf{P}_{r}$ and $\mathbf{Q}_{r}$.

$$
\begin{aligned}
& \mathbf{P}_{r}:=\left[\begin{array}{llll}
\mathbf{B} & \mathbf{A B} & \cdots & \mathbf{A}^{r-1} \mathbf{B}
\end{array}\right] \\
& \mathbf{Q}_{r}:=\left[\begin{array}{llll}
\mathbf{C}^{T} & \mathbf{A}^{T} \mathbf{C}^{T} & \cdots & \left(\mathbf{A}^{T}\right)^{r-1} \mathbf{C}^{T}
\end{array}\right]^{T}
\end{aligned}
$$

Through the eig(29)-eig(32), we can get

$$
\mathbf{H}_{r}=\mathbf{H}\left[\begin{array}{c}
\mathbf{I}_{r} \\
0
\end{array}\right]=\mathbf{Q P}\left[\begin{array}{c}
\mathbf{I}_{r} \\
0
\end{array}\right]=\mathbf{Q} \mathbf{P}_{r}
$$

$\mathbf{h}_{r+1}=\mathbf{H} e_{r+1}=\mathbf{Q P} e_{r+1}=\mathbf{Q} \mathbf{A}^{T} B$

The next step is to solve the problem of the least squares [17].

$\mathbf{H}_{r} x_{l s}=\mathbf{h}_{r}+\min _{x \neq 0}\left\|\mathbf{H}_{r} x-\mathbf{h}_{r}\right\|_{2}$

With the $\min _{x \neq 0}\left\|\mathbf{H}_{r} x-\mathbf{h}_{r}\right\|_{2}$ is the least square error.

For a discrete control system meets the Gramian observable matrix.

$\boldsymbol{\delta}=\mathbf{Q}^{T} \mathbf{Q}$

Which can calculated

$\mathbf{x}_{l s}=\left(\mathbf{H}_{r}^{T} \mathbf{H}_{r}\right)^{-1} \mathbf{H}_{r}^{T} \mathbf{h}_{r+1}=\left(\mathbf{P}_{r}^{T} \boldsymbol{\delta} \mathbf{P}_{r}\right)^{-1} \mathbf{P}_{r}^{T} \boldsymbol{\delta} \mathbf{A}^{r} \mathbf{B}$

The characteristic polynomial of the reduction system is

$\mathbf{x}_{l s}=\left[\begin{array}{llll}1 & \mathbf{z} & \cdots & \mathbf{z}^{r}\end{array}\right]\left[\begin{array}{c}x_{l s} \\ 1\end{array}\right]$

For the reduced order model of the system by using the method of projection

$\boldsymbol{\Sigma}_{l s}=\left[\begin{array}{cc}\mathbf{Z}^{T} \mathbf{A V} & \mathbf{Z}^{T} B \\ \mathbf{C V} & 0\end{array}\right]$ 
Where $\mathbf{Z}^{T}=\left(\mathbf{P}_{r}^{T} \boldsymbol{\delta} \mathbf{P}_{r}\right)^{-1} \mathbf{P}_{r}^{T} \boldsymbol{\delta}, \quad \mathbf{V}=\mathbf{P}_{r}$. The $\mathbf{Z}$ can be calculated by comprising seeking Grames of SVD method [18-19] and on the calculation of the $\mathbf{V}$ can reflect the matching concept. This method can guarantee the matrix of linear combination of $r$ column with the first $r+1$ columns of Hankel singular value has the minimum error ${ }^{[20]}$. Using the method not only can ensure the stability of the reduction model of $r$ order, but ensure that order-reduction system can exactly match the first $r$ with the original model.

\section{Model Reduction Example}

In order to verify the SVD Krylov order reduction method, the Russia international space station model used as benchmarks [21]. The full-order model represents the component1R (Russian Service Module) for the ISS. It is comprised of 270 states, 3inputs and 3outputs [22]. The frequency responses of the MIMO system are shown in the Figure 1.

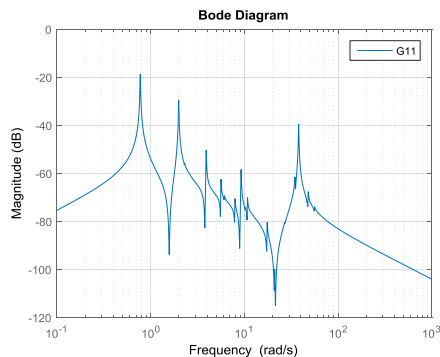

(a)

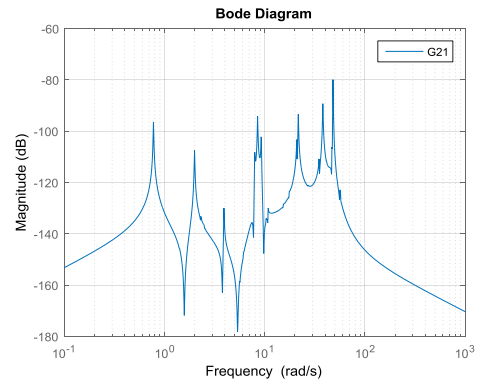

(d)

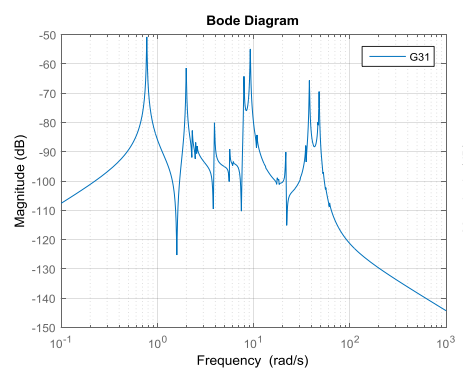

(g)

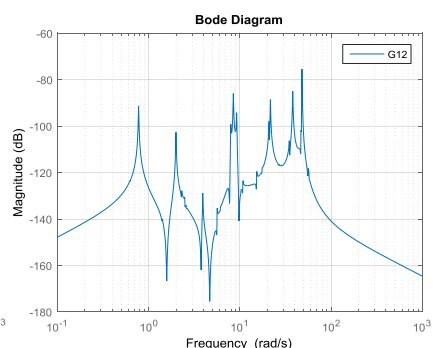

(b)

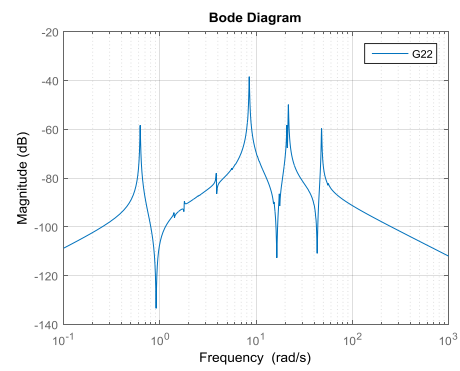

(e)

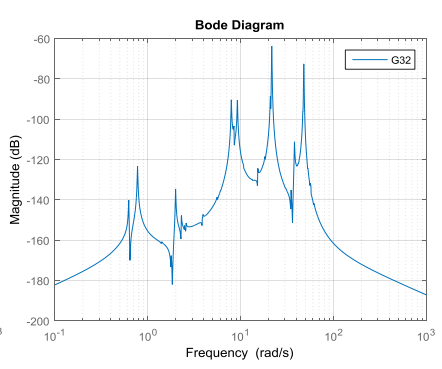

(h)

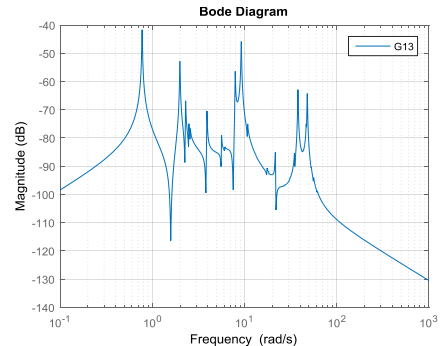

(c)

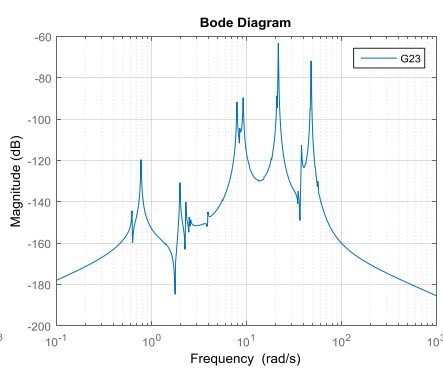

(f)

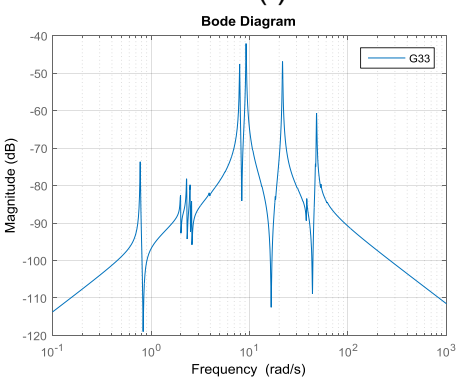

(i)

Figure 1. MIMO Frequency Responses of the 1R Module of the Iss

The matrix A that is given by Iss model is a sparse matrix, the figure of sparse matrix A is shown in Figure 2. The normalized Hankel singular values are shown in Figure 3 . When the system is reduced to 20 order can keep the characteristics of the original system, which can make sure the order reduction system can approximation with the original system. 


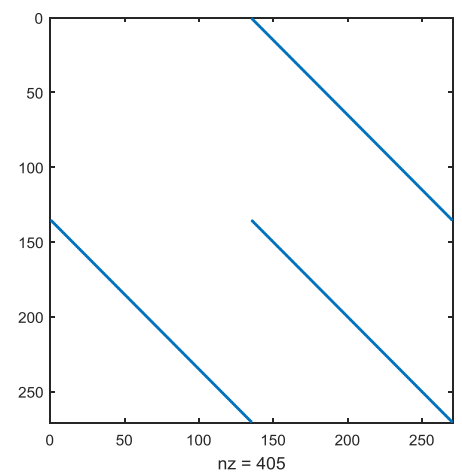

Figure 2. Sparsity of A

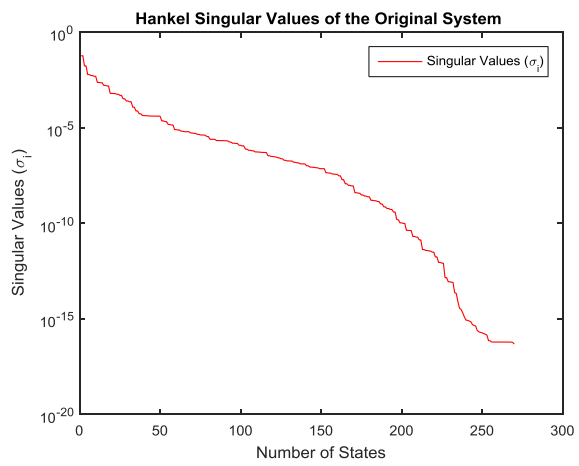

Figure 3. Normalized Hankel Singular Values of the Iss

Using the method of SVD-Krylov, the Iss model are reduced to 120, 50, 20 order. The eigenvalues distribution of the original and the reduced-order model are shown in Figure 4. From the Figure 4, can be seen that the system reduced to 20 order will still be able to better retain most of the original system performance.

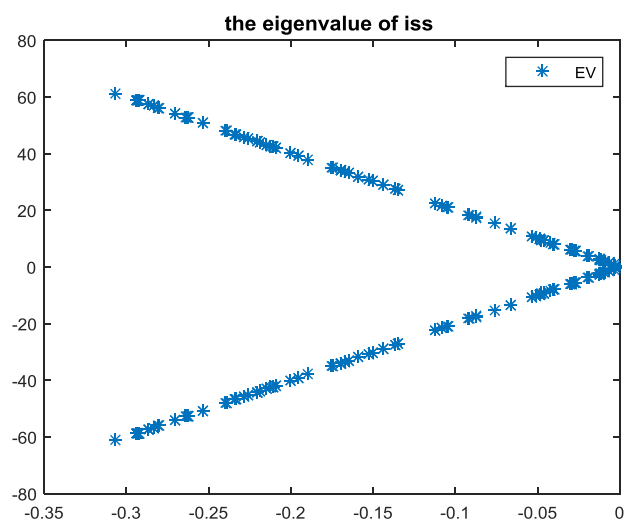

(a)

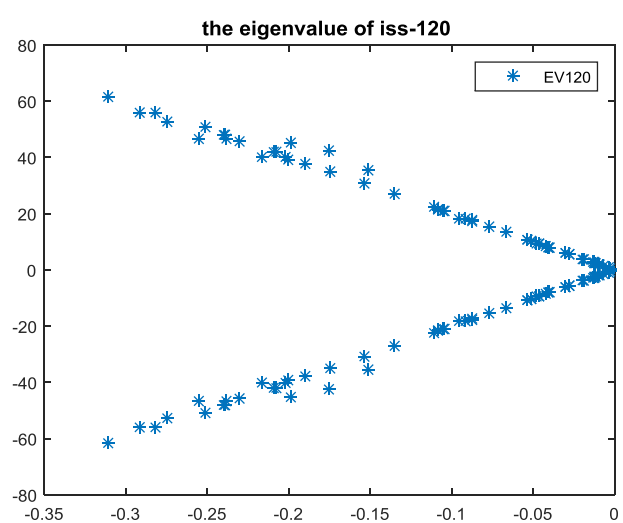

(b) 


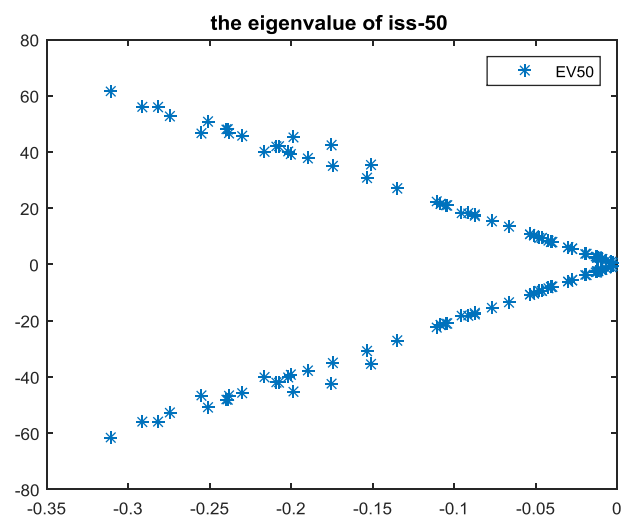

(c)

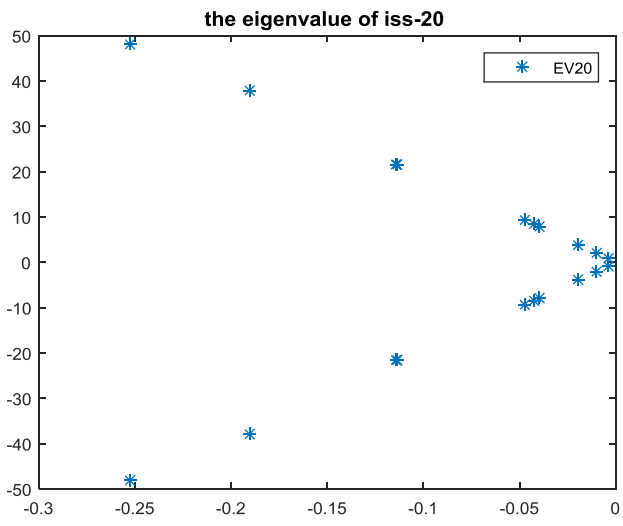

(d)

Figure 4. Eigenvalues of the Original and Reduced-Order Iss

The frequency-response curves of the original and reduced-order of the Iss model are depicted in Figure 5. Through the figure can determine when the system after the order reduction to 10 order, major changes have taken place in the performance of the system, there are great differences with the original system. when the $r=20$ can keep most of the original system performance and satisfies, taht can meet the requirements of order reduction. The system in the low frequency and high frequency has less volatile, a reduced order model in volatile in the frequency and the original model of frequency response is larger difference, the spectrum of especially is appeared after dropped to 10 order, the differences are obvious, so the order reduction effect is not ideal.

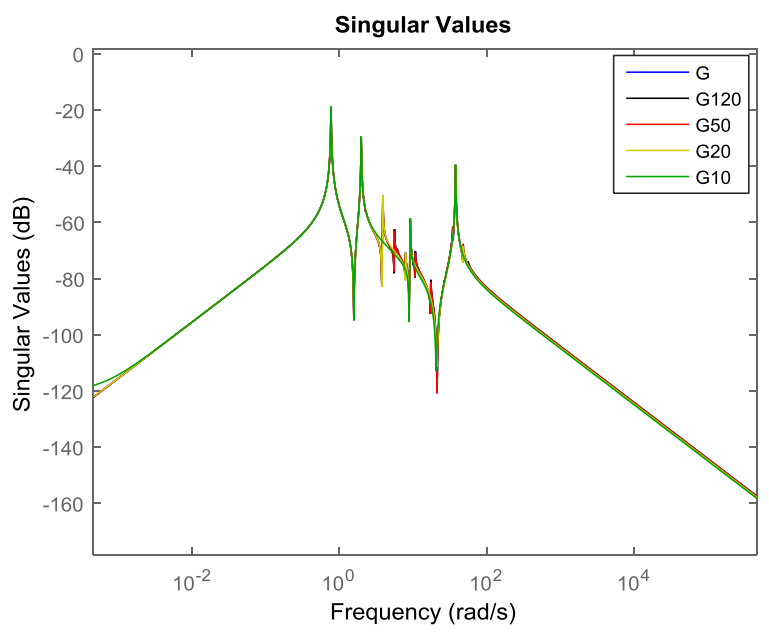

Figure 5 .The Frequency-Response of the Original and Reduced-Order of Iss

The error bound of reduced-order are depicted in Table 1. When $r \geq 20$ have the smaller error bound, with the order of model is lower, the error bound is higher. The performance are not similar with the original model when $\mathrm{r}=10$. 
Table 1. The Error Bound of Reduced-Order Iss

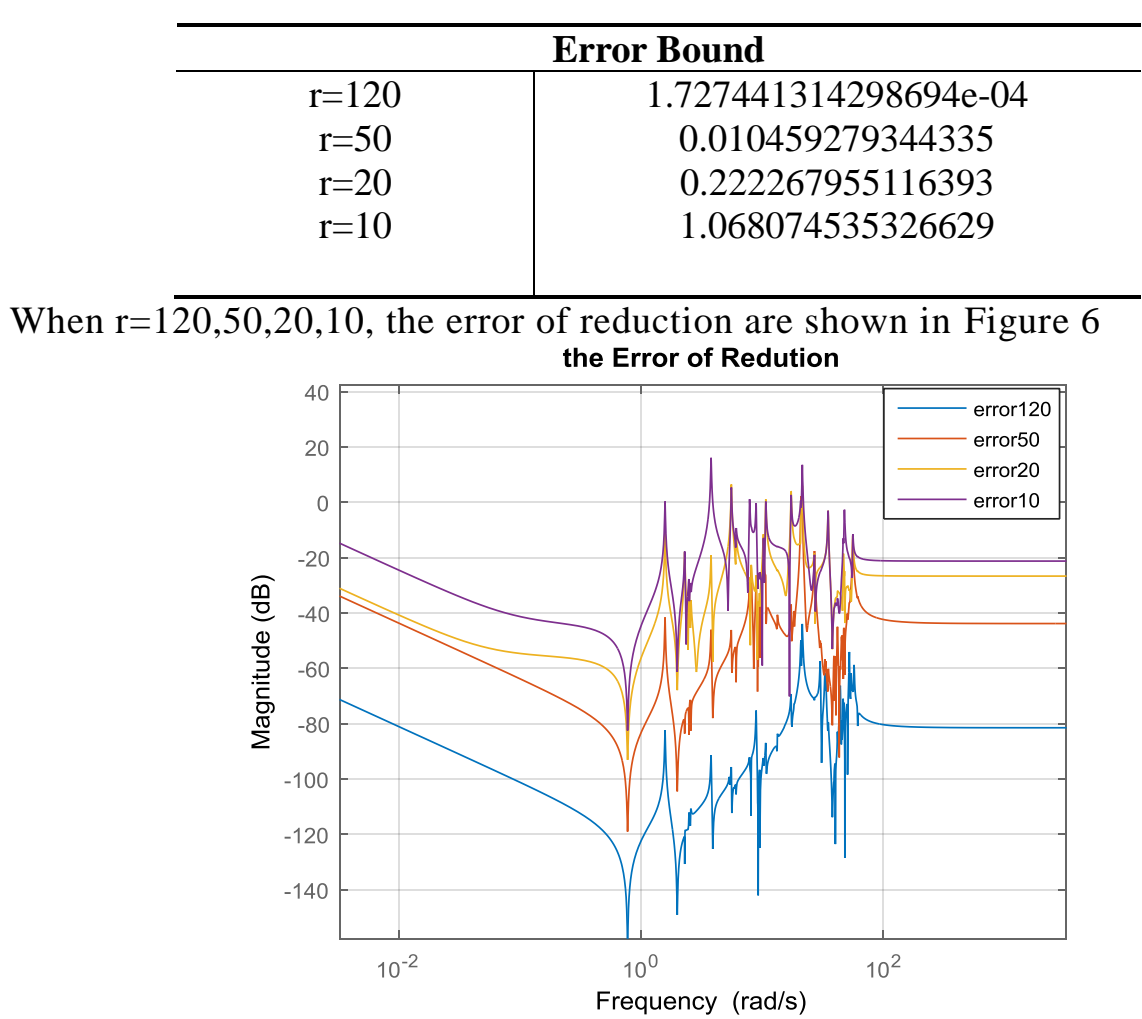

Figure 6. The Error of Reduction

The error is relatively larger when reduced to 10 order. The order greater than or equal to 20, the error is relatively smaller. In the intermediate frequency, the error has the larger change because of the frequency response has relatively larger volatile, the smaller size of model, the larger error.

\section{Conclusion}

We have proposed a model reduction algorithm which combines the SVD and Krylov-based methods. It is a two-sided projection method where one side carries the SVD (Gramian) information and another side the Krylov information. The reduced model matches the first $r$ Markov parameters of the full order model. Through error analysis, it was found that the reduced system turned out to minimize a weighted error. In order to verify the SVD-Krylov order reduction method, we reduced the Russia the international space station model. When the $r=20$ can keep most of the original system performance and satisfies. But when the system reduced to 10 order, major changes have taken place in the performance of the system and have great difference with the original system through the frequency-responses of the original and reduced-order can determine. The results show that the proposed method can improve the large system model order reduction effect which can be widely used in reducing large scale MIMO systems.

\section{References}

[1] E. De Tuglie, S. M. Iannone and F. Torelli, "A coherency recognition based on structural decomposition procedure”, Power Systems, IEEE Transactions on, vol. 23, no. 2, (2008), pp. 555-563.

[2] N. Martins, F. G Silva and P. C. Pellanda, "Utilizing transfer function modal equivalents of low-order for the design of power oscillation damping controllers in large power systems", IEEE PES General 
Meeting, (2005), pp. 2642-2648.

[3] J. J. Sanchez-Gasca and J. H. Chow, "Computation of power system low-order models from time domain simulations using a Hankel matrix", Power Systems, IEEE Transactions on, vol. 12, no. 4, (1997), pp. 1461-1467.

[4] P. D. Olivier, “A comparison of reduced order model techniques”, System Theory, 2009. SSST 2009. 41st Southeastern Symposium on. IEEE, (2009), pp. 240-243.

[5] R. W. Freund, "Model reduction methods based on Krylov subspaces", Acta Numerica, vol. 12, (2003), pp. 267-319.

[6] Y. Lin, L. Bao and Y. Wei, "A model-order reduction method based on Krylov subspaces for MIMO bilinear dynamical systems", Journal of Applied Mathematics and Computing, vol. 25, no. 1-2, (2007), pp. 293-304.

[7] J.Yaolin, "Model order reduction method", press, (2010), pp. 109-152.

[8] A. C. Antoulas, "A new result on positive real interpolation and model reduction", Systems Control Lett, (2005), 54, 361-374.

[9] X. Gang and Y. Chao, "Balanced truncation method in the application of pneumatic servo elastic system model order reduction", Journal of aviation, vol. 22, no.2, (2001), pp. 168-170.

[10] Z. Hongshan, S. Guowei and J.Quanyuan, "Using the theory of balance of power system model order reduction", Transactions of China Electrotechnical Society, vol. 25, no. 2, (2010), pp. 127-133.

[11] T. Reis and T. Stykel, "A survey on model reduction of coupled systems", Model order reduction: theory, research aspects and applications. Springer Berlin Heidelberg, (2008), pp. 133-155.

[12] A. Antoulas, D. Sorensen and K. A. Gallivan, "Model reduction and real-time control for dynamic data druven systems", Dynamic data driven simulation, Ed. F.Darema, Kluwer Publication, (2005), pp. 56-89.

[13] R. V. Polyuga and A. Van der Schaft, "Structure preserving model reduction of port-Hamiltonian systems by moment matching at infinity", Automatica, vol. 46, no. 4, (2010), pp. 665-672.

[14] H. Minghua and H. Shousong, "The balanced order reduction method and its progress", Journal of nanjing university of aeronautics and astronautics, (1990), pp. 15-86.

[15] S. Gugercin and A. C. Antoulas, "A survey of model reduction by balanced truncation and some new results", International Journal of Control, vol. 77, no. 8, (2004), pp. 748-766.

[16] A. C. Antoulas, C. A. Beattie and S. Gugercin, "Interpolatory model reduction of large-scale dynamical systems”, Efficient Modeling and Control of Large-Scale Systems. Springer US, (2010), pp. 3-58.

[17] W. Ligang and H.Yueming, “ $H_{\infty}$ model reduction for linear differential repetitive processes", Control and Decision, vol. 23, no. 10, (2008), pp. 1196-1200.

[18] V. Druskin, L. Knizhnerman and M. Zaslavsky, "Solution of large scale evolutionary problems using rational Krylov subspaces with optimized shifts", SIAM Journal on Scientific Computing, vol. 31, no. 5, (2009), pp. 3760-3780.

[19] S. Gütte, "Rational Krylov approximation of matrix functions: Numerical methods and optimal pole selection", (2012), pp. 10-39.

[20] S. Gugercin and A. C. Antoulas, "Model reduction of large-scale systems by least squares", Linear algebra and its applications, vol. 415, no. 2, (2006), pp. 290-321.

[21] M. R. Akella and R. G. Longori, "Model and controller reduction of large-scale structures based on projection methods", The University of Texas at Austin, (2006), pp. 74-84.

[22] B. Yan, S. X. D. Tan and J. Fan, "Passive rational interpolation-based reduction via Carathéodory extension for general systems", Circuits and Systems II: Express Briefs, IEEE Transactions on, vol. 57, no. 9, (2010), pp. 750-755.

\section{Author}

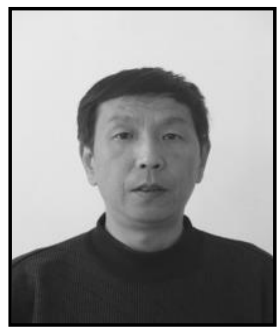

Zhe Yan is a professor at the department of automation from Harbin University of Science and Technology. His research area mainly includes control theory and applications. E-mail: yanzhehrb@163.com 